\title{
Corela
}

Cognition, représentation, langage

HS-8 | 2010

L'interpellation

\section{Les interpellations des professeurs dans l'interaction didactique. Étude comparative dans le domaine français-roumain}

\section{Valentina Barbu}

\section{CpenEdition}

Journals

Édition électronique

URL : http://journals.openedition.org/corela/1587

DOI : $10.4000 /$ corela. 1587

ISSN : 1638-573X

Éditeur

Cercle linguistique du Centre et de l'Ouest - CerLICO

\section{Référence électronique}

Valentina Barbu, «Les interpellations des professeurs dans l'interaction didactique. Étude comparative dans le domaine français-roumain », Corela [En ligne], HS-8 | 2010, mis en ligne le 17 février 2014, consulté le 10 décembre 2020. URL : http://journals.openedition.org/corela/1587 ; DOI https://doi.org/10.4000/corela.1587

Ce document a été généré automatiquement le 10 décembre 2020.

\section{cc) (i) (2) (2)}

Corela - cognition, représentation, langage est mis à disposition selon les termes de la licence Creative Commons Attribution - Pas d'Utilisation Commerciale - Partage dans les Mêmes Conditions 4.0 International. 


\title{
Les interpellations des professeurs dans l'interaction didactique. Étude comparative dans le domaine français- roumain
}

\author{
Valentina Barbu
}

\section{Introduction}

1 L'idée qui sous-tend cette étude est que la production et l'interprétation des interpellations sont soumises à des contraintes linguistiques et situationnelles. Une description pertinente de ces unités devrait s'inscrire dans le champ de l'analyse des interactions authentiques. Les deux buts poursuivis dans ce travail sont, l'un, plus général - celui de fournir une description fonctionnelle aux interpellations dans l'interaction scolaire, l'autre, plus particulier - celui d'étudier les variations linguistiques des interpellations en français et en roumain en tant que langues maternelles.

2 Après une partie introductive dans laquelle est défini le cadre théorique de l'analyse, une deuxième partie traite des principales réalisations linguistiques des interpellations dans les deux langues susmentionnées, produites par les enseignants dans le cadre de l'interaction en classe. Enfin, une troisième partie propose une synthèse comparative des réalisations linguistiques des interpellations dans le domaine français / roumain afin de mettre en évidence les caractéristiques de ces unités linguistiques dans chacune des langues visées.

\section{Méthodologie et corpus}

3 La démarche repose sur l'analyse comparative des unités mentionnées dans des classes de français et de roumain en tant que langues maternelles. Pour que la comparaison soit 
valide, nous avons choisi le même niveau scolaire, le CE1, dans les deux systèmes d'enseignement, français et roumain, et nous avons effectué le même nombre d'heures d'enregistrements dans les classes observées pendant plusieurs semaines, soit huit heures pour chaque langue observée. Le corpus a été étudié en fonction d'une grille d'analyse qui vise l'aspect linguistique (choix des réalisations syntaxiques des interpellations, les "schèmes interpellatifs " des professeurs, interpellation et actes illocutoires, interpellation et termes d'adresse, interpellation et modalités énonciatives, structure des échanges et des interventions) et situationnel (univers de référence, situation de l'interaction, participants à l'interaction). Dans cet article, nous allons présenter les résultats de l'étude du corpus sans séparer les domaines isolés supra. Cette approche intégrée nous montre leur fonctionnement indissociable pour une description approfondie de l'interpellation.

\section{Acception et description du terme « interpellation »}

4 «Apostropher ", " appeler ", "adresser la parole brusquement à quelqu'un ", « questionner (un suspect) sur son identité » (droit pénal), « demander à un ministre ou à un gouvernement de s'expliquer sur ses actes » (politique) (Le Robert, 2007), acte allocutif (Charaudeau, 1992), forme d'intimation (Benveniste, 1974), opération discursive interpersonnelle (dans les analyses des conversations), etc., ou tout cela à la fois, l'interpellation ne se laisse pas facilement décrire.

5 2.1. Dans cette étude, l'interpellation sera traitée comme un acte métadiscursif dont le rôle est d'assurer les conditions normales de déroulement du discours/des actes de discours proprement dits. En outre, il vise la sélection du prochain interlocuteur. C'est l'hypothèse théorique proposée par A. Măgureanu (2008) qui définit la classe des actes/discours métadiscursifs comme une pratique sémiotique, une modalité particulière d'utilisation d'un système de signes ${ }^{1}$. L'auteur propose une sous-classification de cet ensemble d'actes : l'interpellation appartiendrait à la sous-classe d'actes/discours phatiques « dont le but est l'établissement, le maintien ou la suspension de la relation interlocutive» (209). A la lumière de cette hypothèse, l'interpellation est un acte métadiscursif qui institue l'interlocuteur. Retenons également que l'interpellation est « un discours qui 'parle' d'un autre discours » (idem).

6 2.2. Le terme " interpellation » se laisse difficilement définir, comme nous le montre la variété des définitions et des approches que nous avons mentionnées au \$2, à cause du fait que c'est une unité/catégorie en lien avec d'autres catégories en linguistique comme les termes d'adresse, les pronoms personnels de $2^{\text {e }}$ personne, les interjections, les impératifs, etc.

$7 \mathrm{Vu}$ le caractère spécifique de notre corpus, nous retenons pour cette étude une description qui met l'accent sur la relation interlocutive. Ainsi le terme peut être paraphrasé par "adresser la parole à quelqu'un pour lui demander ou lui dire quelque chose ». Par ailleurs, dans les classifications des actes de langage, "appeler " représentatif de l'interpellation dans son sens étymologique, a été retenu deux fois par Searle (1979/1982) et une fois par Katz (1977).

8 2.3. Acte métadiscursif, l'interpellation est le plus souvent intégrée à une intervention. En ce sens, elle peut être combinée avec un acte de langage, comme dans l'intervention reproduite sous (1) - où l'interpellation est un acte subordonné par rapport à l'acte 
directeur-, ou on a affaire à deux actes métadiscursifs - «interpellation" et « allocation de la parole » -, comme dans l'exemple (2):

(RLM) Spune, Adi. ${ }^{2}$ (fr. « Dis-nous, Adi. »)

(FLM) A toi, Théophile.

Dans l'énoncé reproduit sous (1), l'interpellation est un acte subordonné par rapport à l'acte directeur. Pour examiner de plus près les liens entre l'interpellation et les actes de langage d'une part, et entre le comportement de cette catégorie naturelle en contexte authentique d'autre part, nous allons procéder par la suite à l'analyse des données du corpus.

\section{Analyse du corpus. Les réalisations linguistiques des interpellations}

10 Comme le montre la variété d'approches dont elle fait partie, l'interpellation interfère avec plusieurs catégories en linguistique que nous allons analyser par la suite afin d'étudier le comportement fonctionnel de l'interpellation en contexte. $\mathrm{Vu}$ la complexité du sujet, il est évident que nous ne pourrons pas recouvrir tous les domaines connexes à l'interpellation mais seulement ceux qui présentent un intérêt particulier pour le type de contexte étudié, à savoir l'interaction scolaire.

\subsection{Interpellation et actes de langage}

11 Revenant sur la description de l'interpellation, nous avons établi qu'elle est un acte métadiscursif. C'est pour cette raison que dans ce sous-chapitre nous traiterons de l'interpellation et des actes de langage. Par la suite, nous allons présenter les combinaisons dans lesquelles elle entre avec les différents actes illocutoires.

Avant de passer à la présentation de cette situation précise, il convient premièrement de décrire un autre terme qui joue un rôle important dans cette analyse, à savoir l' « acte de langage ». Dans cette étude, un acte de langage est constitué d'une force illocutoire appliquée à un contenu propositionnel. (Cf. Searle, 1972, 1982, Searle \& Vanderveken, 1985, et Vanderveken, 1988). Nous allons opérer donc avec les classes d'actes de la taxinomie de Searle (1972) et décrites succinctement dans la citation suivante ${ }^{3}$ :

«Nous disons à autrui comment sont les choses (assertifs), nous essayons de faire faire des choses à autrui (directifs), nous nous engageons à faire des choses (promissifs), nous exprimons nos sentiments et nos attitudes (expressifs) et nous provoquons des changements dans le monde par nos énonciations (déclarations). » (32)

Pour l'analyse des actes de langage en contexte, nous adoptons la démarche proposée par le Groupe de Recherches sur les Communications de l'Université de Nancy 2, et théorisée par Christian Brassac et Alain Trognon (Cf. Brassac, 1992, ou Trognon et Brassac, 1992). En prenant comme point de départ la logique illocutoire développée par Searle \& Vanderveken (1985) et plus tard par Vanderveken $(1988,1990)$ qui prend comme composants primitifs non pas les actes de langage mais les composants de la force illocutoire, le modèle ainsi développé permet de dériver un ensemble de relations logiques articulant les propriétés des actes de langage. La logique illocutoire de la théorie des speech acts, entendue comme logique des relations entre réussite et satisfaction entre les actes de langage, n'est pas prise à la lettre dans l'approche susmentionnée. D'un côté, 
les tenants de ce modèle étendent la portée de certaines lois de cette logique, et de l'autre elle est interprétée dans un modèle dialogique face au modèle monologique initial. Les analyses faites au sein du GRC ont mis en évidence «le jeu croisé des conditions de satisfaction d'un acte relativement à la réussite d'un autre acte avec lequel il est en relation » (Brassac, $1992: 68$ ). Pour expliquer cette approche, il nous semble important de reprendre schématiquement l'analyse d'un échange réparateur (cf. Trognon, 1991a, 16-17 et 1991e, 224-225, apud Brassac, 1992: 69):

L1 Quelle heure est-il?

L2 Huit heures.

L1 Merci.

14 En suivant la logique illocutoire classique des actes de langage nous identifions le premier acte comme directif, et le deuxième comme assertif. Mais l'analyse des chercheurs nous donne des explications plus complexes. Ainsi le second acte illocutoire, l'assertif, satisfaitil le premier acte illocutoire, le directif. Plus précisément, le locuteur L2 répond à la demande en ajustant le monde aux mots par le fait qu'il donne le renseignement demandé. Ce second acte entraîne l'accomplissement réussi du premier, en vertu du fait que la satisfaction d'un acte dont la direction d'ajustement va du monde aux mots implique sa réussite. Donc «il [ce deuxième acte] fixe la valeur communicative du premier énoncé. » (Brassac, op. cit., p. 69). C'est la satisfaction du premier acte, lors du deuxième tour de parole, qui permet son accomplissement: "l'acte de langage rétrospectivement accompli via l'intervention réactive est l'acte de langage dont le contenu propositionnel représente les conditions qui ont été satisfaites lors de l'intervention réactive » (Trognon, apud Brassac, idem). En effet, le modèle esquissé cidessus rend compte de la mise en séquence des actes de langage en conversation, ce qui amène les chercheurs du GRC à conclure qu'il est possible de relier théorie des actes de langage et théorie des séquences conversationnelles.

15 L'acte de langage est un acte qui agit à deux niveaux d'analyse : le discours oral et le texte ${ }^{4}$. C'est pour cette raison que nous utilisons aussi un modèle hiérarchique à cinq rangs qui puise dans le modèle genevois (Cf. Roulet et al. 1985), en changeant sensiblement les deux premiers termes en fonction du spécifique de notre corpus, ce qui donne les unités suivantes: l'interaction (le cours de langue maternelle), la séquence, l'échange, l'intervention et l'acte de langage. En outre, dans notre modèle la force illocutoire appartient aux actes de langage, et non aux interventions, comme dans le modèle susmentionné, parce qu'il existe des interventions qui sont constituées de deux ou plusieurs actes de langage qui ont des forces illocutoires différentes, comme nous allons le montrer dans cette étude.

Deuxièmement, nous voudrions souligner l'importance de distinguer entre la forme et le contenu des actes de langage, c'est-à-dire entre la valeur illocutoire de l'acte et le type de phrase par lequel il est exprimé. Dans son ouvrage sur l'apostrophe nominale (2007), Catherine Détrie remarque le fait que l'interpellation peut s'effectuer dans des énoncés dont la modalité peut être injonctive - «le mode d'ancrage le plus fréquent de l'apostrophe » (19) - interrogative ou assertive.

17 Dans ce qui suit nous allons classer les énoncés interpellatifs, en fonction d'une part du type de modalité qui lui est associée, et de l'autre de la personne grammaticale corrélée avec le terme qui désigne l'interpellé. Ces opérations nous donnent les premières informations par rapport aux schèmes interpellatifs des professeurs. 


\subsubsection{Interpellation et actes de langage directifs :}

18 Nous avons déjà vu dans la description des actes de langage de Searle (voir supra 3.1.) que le but illocutoire de la classe des directifs est de «[faire] faire des choses à autrui ». Ainsi parmi les actes directifs identifiés dans l'interaction didactique, mentionnons-nous la requête et la question, deux réalisations d'un macroacte - la demande -, le premier visant le faire et le deuxième le dire. Avant de continuer, une précision terminologique : nous considérons comme requête tout énoncé produit par un locuteur pour demander à son interlocuteur d'accomplir un acte quelconque (verbal ou non verbal) 5 .

19 a) Modalité injonctive et deuxième personne du singulier ou pluriel coréférente avec la personne interpellée :

(3) (FLM) Paul Augustin !

(4) (RLM) Petrică, fii atent ! (fr. « Pierre, fais attention! »)

20 En ce qui concerne sa réalisation linguistique, ce type d'énoncé est réduit le plus souvent au terme qui désigne l'interpellé, comme dans l'exemple (2) du FLM. Pour pouvoir interpréter correctement cet énoncé, en tant que directif - demande de se taire -, il faut le situer dans son contexte de production.

21 b) Modalité interrogative et deuxième personne corrélée avec la personne interpellée :

(5) (FLM) Est-ce que tu as fini, Raphaël ?

(6) (RLM) Deranjezi colegul, Ruxi? (fr. «Tu déranges ton camarade, Ruxi ?»)

Dans le deuxième exemple, il ne s'agit pas d'une question mais d'une requête, d'un rappel à l'ordre.

c) Modalité assertive et troisième personne du singulier ou pluriel coréférente à la personne interpellée :

(7) (FLM) C'est Sixtine qui va commencer à lire.

(8) (RLM) Alexandra, copii, ne face un model la tablă ${ }^{6}$. (« Alexandra, les enfants, va nous montrer un exemple au tableau. »)

Nous remarquons dans ces exemples une situation peu habituelle pour l'interpellation : la corrélation du terme qui désigne l'interpellé avec la $3^{\mathrm{e}}$ personne (du singulier, dans ces exemples précis). Qu'est-ce qui nous permet de dire que ces termes désignent véritablement les interpellés? Tout comme dans le cas de l'analyse des actes de langage, le contexte et le cotexte désambigüisent l'interprétation. Dans les deux cas susmentionnés, Sixtine et Alexandra ratifient à la fois les actes de langage directifs ${ }^{7}$ - requête en contexte didactique -, et les termes qui désignent les interpellés, par leurs réactions lors du deuxième tour, qui n'est pas un tour verbal mais non verbal. Ainsi Sixtine commence-t-elle à lire et Alexandra va-t-elle au tableau pour dessiner le modèle. Donc la réaction de l'allocutaire fonctionne donc comme un test pour l'identification du terme qui désigne l'interpellé.

Sachant que le terme qui désigne l'interpellé est corrélé dans la majorité des cas avec un pronom de $2^{\text {e }}$ personne - notre corpus étaye cette observation - nous postulons que les énoncés de ce type représentent des interpellations indirectes. Pour étayer une telle observation, nous citerons la description des actes indirects donnée par Searle (1982) : «des actes de langage indirects dans lesquels un acte illocutoire est accompli indirectement par l'accomplissement d'un autre acte illocutoire»(72). Son analyse décompose un énoncé comme « Peux-tu me passer le sel? » en acte illocutoire primaire - demande (de faire quelque chose) - et acte illocutoire secondaire - question. En 
appliquant ce type d'analyse à un de nos exemples - (4) -, nous décelons les interpellés suivants : Comme le remarque Lagorgette (2006) ce phénomène " reste difficile à analyser à l'aune des critères usuels : il n'appartient pas aux parties du discours, peut en mêler plusieurs en sa composition, peut fonctionner de manière autonome, et de fait semble faire fi des plus élémentaires règles de syntaxe puisqu'il se promène d'un bout à l'autre des énoncés sans place assignée ". Ces aspects ont donné naissance à un foisonnement de définitions, de descriptions du segment en cause en fonction aussi de l'approche à l'intérieur de laquelle il a été traité. La notion de terme d'adresse (TA dorénavant) a été élaborée par des ethnologues et anthropologues. Le choix de cette appellation met l'accent sur le caractère pragmatique des termes concernés dans le discours. Dans la présente étude le TA s'applique à toute forme renvoyant dans le discours à l'autre, soit directement (valeur allocutive) soit indirectement (valeur délocutive).

La présence d'un TA est " un indice explicite de l'interpellation », comme le remarque Détrie $(2007,19)$. Par la suite, nous allons faire premièrement un inventaire succinct des TA dans les deux langues, et ensuite nous allons mentionner les valeurs de ces unités linguistiques pour conclure sur les similitudes et les différences en français et en roumain. Aussi traiterons-nous tout d'abord des énoncés où les interpellés sont nommés explicitement par des termes d'adresse ${ }^{8}$.

\subsubsection{Inventaire des TA}

En ce qui concerne les termes qui désignent les interpellés dans l'interaction didactique nous avons d'une part les pronoms (personnels, indéfinis) et de l'autre les noms personnels. 

personnels ( $1^{\text {ère }}$ et $2^{\mathrm{e}}$ personne, singulier/ pluriel), soit les pronoms indéfinis («tous", " quelqu'un d'autre »). Les emplois ne sont pas les mêmes car les premiers font partie de ce que nous appelons "requête à adresse individuelle" (voir infra § 3.4.1.) vu le rôle déictique des pronoms personnels de première et deuxième personne, tandis que les seconds entrent dans les « requêtes à adresse collective ».

(12) (RLM)

(a) P Da. Spune tu prima. (fr. « Oui. Toi la première. »)

(b) P Miruna, hai şi tu mai repede un pic. (fr. « Allez, Miruna, allez un peu plus vite

toi. »)

(13) (RLM)

(a) P Ce fac eu în fiecare dimineaŢă ? Eu şcolarul? (fr. « Qu'est ce que je fais moi, chaque matin? Moi, l'écolier?»)

(b) P Hai, Ioana, să scriem. (fr. « Allez, Ioana, on écrit. »)

(14) (FLM)

(a) P A toi, Théophile.

(b) P Comment ça se fait que tu les aies pas faites, Louis ?

(15) (FLM)

P On ne dérange pas les enfants qui sont encore en train de travailler. ((en s'adressant à un élève qui faisait du bruit))

Nous commençons notre analyse par les pronoms personnels car plus nombreux. À la différence $d u$ français, en roumain le pronom personnel sujet n'est pas obligatoire, ce qui explique le nombre important d'énoncés où le terme d'adresse n'est pas explicite. La présence explicite de ce pronom marque une sorte de "violation » du bon déroulement de la classe. Dans l'énoncé de l'exemple (12), à travers le directif, le professeur exprime aussi son mécontentement par rapport au comportement de l'élève. Un autre marqueur qui entre en jeu est de nature suprasegmentale. Dans ce cas, on remarque une scalarité dans la formulation des interpellations exprimée par la hauteur de la fréquence fondamentale des TA et aussi par le fait que ce segment est frappé par un accent d'insistance. Comparons dans ce sens l'énoncé reproduit en (12) (a) qui est neutre parce qu'il marque juste la sélection du futur locuteur à l'énoncé (12) (b) qui est marqué. Une situation pareille est présente en français (voir les exemples donnés sous (13).

En français, la situation est moins évidente, vu que le pronom sujet est obligatoire. Dans ce cas, on remarque la présence d'autres marqueurs, qui sont surtout de nature paraverbale, l'intonation et l'accent.

Une situation intéressante est illustrée en (13 a) où l'on remarque la présence du pronom personnel de première personne. Celui-ci marque formellement un emploi autodésignatif mais en réalité c'est un emploi appellatif. On voit bien que, même si c'est le professeur qui parle, le référent n'est pas lui, mais chaque personne qui fait partie de la classe d'élèves. D'ailleurs, l'ambigüité est levée lors du deuxième énoncé où le référent du pronom « eu » (fr. « moi ») est explicité par le mot " écolier ». En outre, l'emploi explicité de ce pronom est lié à la présence de la requête à adresse collective. En (13 b) nous avons une autre situation d'emploi du pronom personnel de première personne. En roumain, le TA «Ioana» est corrélé avec la première personne du pluriel, marque de l'emploi hypocoristique. En français, la situation parallèle serait l'utilisation du pronom " on », ce qu'on voit dans la traduction en roumain mais aussi dans l'exemple (15). L'analyse du corpus RLM a mis en évidence un nombre beaucoup plus important d'énoncés où le pronom personnel acquiert une telle valeur face au corpus FLM. Ces données étayent une observation faite en 1959 (1976) par Valeriu Rusu qui mentionne l'importance du facteur 
affectif dans les réalisations linguistiques des formules d'interpellation: «Ce développement des formules d'interpellation, leur variété et leur fréquence, semblent constituer un caractère 'balkanique' de la langue roumaine. [...] L'étude de cet aspect du lexique de la langue roumaine est également intéressante à un autre point de vue : le facteur affectif joue un grand rôle dans cette partie du lexique qui influence particulièrement le moyen de communication linguistique." (243/1) Ce «facteur affectif » est plus évident encore dans les énoncés tirés du corpus RLM où certains TA sont exprimés par des "diminutifs" des prénoms des élèves, comme nous allons le montrer infra. Les réalisations linguistiques des TA représentent aussi une marque de la relation professeur-élève(s).

Comme on le remarque, la transposition en français peut être faite par des pronoms toniques seulement.

D'autres marques explicites de l'interpellation sont les pronoms interrogatif-relatifs et les pronoms indéfinis, toujours dans des requêtes adressées à la classe avant de sélectionner précisément l'allocutaire :

(16) (RLM) Ia să vedem cine? Cine o mai ajută requête à adresse collective ? Cine îmi spune requête à adresse collective ? Paul requête à adresse individuelle. (fr. « Voyons qui ? Qui veut l'aider?

Qui veut répondre? Paul. »)

(17) (FLM) Qui connaît Oscar requête à adresse collective ? Blanche requête à adresse individuelle .

(18) (RLM) Altcineva fără să citească cerinţa requête à adresse collective . Cu cuvântul livadă. Andreea requête à adresse individuelle . (fr. "Quelqu'un d'autre, sans qu'il lise la consigne. En utilisant le mot 'verger'. Andreea. »)

(19) (FLM) Est-ce que quelqu'un peut me dire le nombre du mois de janvier requête à adresse collective ? Sixtine requête à adresse individuelle .

41 Enfin, nous abordons des TA les plus nombreux, les noms personnels (noms de famille ou «patronymes", prénoms, diminutifs et surnoms). C'est un type de TA qui permet de discriminer l'allocutaire et de l'identifier en propre. Nous avons déjà remarqué qu'ils sont présents dans presque toutes les interpellations extraites du corpus, le plus souvent en corrélation avec les pronoms, ce qui nous donne des informations supplémentaires par rapport au type de relation interpersonnelle. Plus précisément, si le TA-prénom se combine avec le pronom " on ", on parle d'un rapprochement dans la relation L1-L2 (ex. (23)), tandis qu'une combinaison TA-prénom + nom de famille avec un pronom de deuxième personne (explicite ou implicite) est l'indice d'une relation interpersonnelle éloignée.

(20) (RLM) Paula şi Andrei Bogza! (fr. « Paula et Andrei Bogza! ») (prénom + nom de famille)

(21) (FLM) Mathieu Quatravaux n'est pas là ? (prénom + nom de famille)

(22) (RLM) Ia să vedem. Ioana. Tu erai. (fr. "Voyons. Ioana. C'était ton tour.» (prénom)

(23) (FLM) On colorie, Léa. (prénom)

(24) (RLM) Altă variantă. Miru, spune. (fr. « Une autre variante. Miru, dis-nous. »)

(diminutif)

(25) (FLM) T'inquiète pas, ma puce, on va régler ça. D'accord ? (terme « affectif »)

(26) (RLM) şi tu, Brutus ${ }^{9}$ ? (fr. « Toi aussi, Brutus ? ») (surnom/emploi ironique)

On mentionne aussi la présence des termes désignant la collectivité-classe, comme « copii » (« les enfants »), « clasa a doua » (« les CE1») en roumain, et « les enfants » en français.

(27) (RLM) Clasa a II-a, ne relaxăm. (fr. « Les CE1, on se calme. »)

(28) (RLM) Copii, vă spun acum că [...] (fr. «Les enfants, je vous dis maintenant 
que $[. .] »$.

(29) (FLM)

(a) Allez, les enfants ! Vous mettez votre tablier.

(b) Quand vous aurez un petit moment de libre, les enfants qui veulent, sur une petite feuille [...] ou sur le cahier d'entraînement, ils pourront écrire la plus belle lettre.

(30) (FLM) Allez, les facteurs ! ou Les distributeurs

À cause du fait que ce type d'organisation manque dans la classe de RLM, on ne retrouve pas de tels TA dans le corpus roumain.

Enfin, on mentionne la présence en roumain de «particules d'interpellation » (Rusu, 1959), comme dans l'exemple suivant :

(31 (FLM) Măi, cine vrei să fie atent când sună soneria? (fr. « [Hé]/ Ø, qui est-ce qui doit faire attention à la sonnerie? »)

Conformément à l'étude de Rusu, le roumain a développé toute une série de formules d'interpellation équivalentes à «tu», mais différenciées sémantiquement. En reproduisant la série sémantique donnée dans cet article (248/6) nous remarquons des degrés toujours plus élevés de politesse :

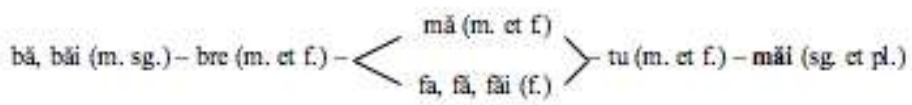

Comme il s'agit de toute une série sémantique en roumain, il est difficile de trouver le terme juste en français, l'interjection « hé » étant peut être un peu trop forte pour la situation présentée.

\subsubsection{Valeurs des TA (allocutive, délocutive)}

Dans les énoncés extraits du corpus nous avons décelé deux valeurs : allocutive (par exemple les énoncés (24) et (25)) et délocutive (exemples (32) et (33)). Notons que le terme qui a la fonction allocutive sert à sélectionner et solliciter un intervenant (dans cette situation précise, un élève) ou plusieurs intervenants (par exemple « les distributeurs ») parmi tous les autres qui sont dans la classe. Le pronom personnel qui lui correspond est celui de deuxième personne. À son tour, la valeur délocutive sert, d'une part, à informer les auditeurs de l'identité du futur intervenant et, de l'autre, à sélectionner et informer en même temps cet intervenant. Le pronom personnel qui lui correspond est celui de troisième personne et on associe cette valeur à l'interpellation indirecte. Nous reviendrons infra sur cet aspect (\$3.4.1.) Pour plus de précision concernant la valeur délocutive, regardons les exemples suivants :

(32) (FLM) C'est Sixtine qui va commencer à lire.

(33) (RLM) Alexandra, copii, ne face un model la tablă. (fr. « Les enfants, Alexandra

va nous montrer un exemple au tableau. »)

En français un autre énoncé où le TA a une valeur délocutive est donné en (29).

\subsection{Distribution syntaxique des termes qui désignent les interpellés}

La place à l'intérieur de la phrase des termes qui désignent les personnes interpellées - les termes d'adresse - nous donne des informations de nature extralinguistique. Ainsi 
pour la distribution syntaxique initiale notons-nous la sélection du destinataire, posé en topique explicite, et à qui le reste du message - en focus - est destiné, comme dans les exemples (34) et (35) :

(34) (RLM) Petrică, fii atent! (fr. 'Pierre, fais attention !')

(35) (FLM) Léa, ça t'intéresse ce qu'elle dit Louise ?

51 Serbat (1996) cité par Détrie $(2006,86)$ affirme que l'apostrophe (dans notre analyse, le TA) à l'initiale de tour ou de phrase correspond à un «acte préparatoire à la communication». Le TA est donc «un marqueur d'ouverture du jeu interlocutif, le syntagme en apostrophe explicitant la ratification de l'allocutaire. » (Détrie, idem)

En ce qui concerne les TA en position médiane, ceux-ci confirment la sélection de l'intervenant (ex. (36)) ou le prolongement de la prise de parole (ex. (37)) :

(36) (RLM) Hai Ioana, să scriem. (fr. « Allez Ioana, on va écrire. »)

(37) (FLM) Répète, Victor, s'il te plaît.

Le nombre réduit des énoncés de cette catégorie pourrait être expliqué par le caractère spécifique de l'interaction étudiée. En classe il est nécessaire d'avoir un nombre important d'élèves qui parlent, ce qui fait que la personne interpellée change assez vite. Comme le remarquait C. Détrie (2006) la fonction interpellative est moins importante que dans le cas de la survenue initiale ou finale parce que la personne désignée par le TA a été déjà ratifiée en tant qu'allocutaire. Ce qui prédomine est une fonction de marquage phatique pour montrer le contact avec la personne interpellée.

54 La distribution syntaxique finale des TA est compatible avec deux fonctions au moins (ce que nous avons décelé dans le corpus étudié) : (i) "ponctuant de clôture » (cf. Détrie, 2006) comme dans l'exemple (38) et (ii) signaler le terme imminent de la prise de parole de l'allocutaire comme on le voit dans l'exemple (39):

(38) (RLM) S-a sunat, copii. (fr. «La sonnerie a retenti, les enfants. »)

(39) (FLM) C'était quand ton anniversaire, Candisse ?

Enfin, le dernier type de distribution syntaxique est constitué par le cas où le TA forme un " énoncé à lui seul », classe d'énoncés qui enregistre une grande fréquence et un panel assez varié de réalisations en fonction des types syntaxiques de phrases avec lesquels il se combine. Regardons les exemples suivants :

(40) (RLM) (a) Toni. / (b) Paula !

(41) (FLM) (a) Léa. / (b) Paul Augustin ! / (c) Paul ?

En ce qui concerne la combinaison avec la phrase assertive, la fonction de l'interpellation est celle de sélection du futur intervenant. Le TA dans une phrase injonctive marque le plus souvent le rappel à l'ordre. Un exemple qui ne se laisse pas facilement interpréter est donné en (41 c). Et cela parce que, pour désambigüiser l'interprétation, on a besoin du contexte. Il s'agit du moment organisationnel où le professeur fait l'appel :

(41c')

(T1) P Paul ? Il est où Paul ?

(T2) E Il n'est pas là.

57 Il s'agit d'une interpellation « apparente » par rapport au référent du TA car on ne peut pas interpeller quelqu'un dont on ne sait pas s'il est présent. En réalité, l'interpellation s'adresse aux autres élèves, comme on le remarque dans l'énoncé qui suit.

Certes, on pourrait toujours imaginer une situation où un TA se combine avec la modalité interrogative en formant un énoncé à lui seul et qui exprime une demande d'accord, par exemple. 

d'une part du contexte et d'autre part du cotexte. Dans la plupart des cas, il fait partie d'un échange à trois interventions, comme on peut le constater :

$\left(40 a^{\prime}\right)$

P Alt răspuns. Toni. (fr. « Autre réponse. Toni. »)

E Din stol câteva rândunele au atins streaşina casei deoarece voiau să-şi ia adio de la cuiburile lor.

(fr. «En guise d'adieu à leurs nids, certaines hirondelles du vol ont touché le toit de la maison.»)

P De la cuiburile lor. Aşa. Foarte bine ! (fr. « À leurs nids. Oui. Très bien ! »)

$\left(41 a^{\prime}\right)$

$P$ Qu'est-ce qu'il y a d'autre chose sur cette lettre ? Léa.

L Chers enfants.

P Ah! Il y a écrit « chers enfants ».

On remarque le fait qu'il est préparé par une pré-requête et que pour arriver à cette autonomie syntaxique le TA-énoncé à lui seul suit un certain parcours à l'intérieur d'un échange.

\subsection{Interpellation, échanges et enchaînement}

\subsubsection{Analyse en unités hiérarchiques}

61 Nous avons mentionné supra (\$3.1.) que, pour l'identification des unités du discours, nous nous appuyons sur un modèle hiérarchique inspiré du premier modèle genevois. Ainsi identifions-nous l'interaction didactique ou le cours de langue, comme première unité du modèle. Celle-ci est constituée de trois séquences, en tant qu'unités immédiatement inférieures hiérarchiquement - incipit, corps de l'interaction et excipit. L'unité suivante, le troisième rang donc, est l'échange qui est constitué à son tour des interventions. Enfin, les interventions sont constituées d'un ou de plusieurs actes de langage. Dans cette étude nous travaillerons surtout avec les trois dernières unités car c'est à ce niveau que l'on localise l'interpellation. Plus précisément le rang-hôte de l'interpellation est l'intervention.

Nous plaidons pour une interprétation contextualisée des unités de discours et c'est pour cette raison que, pour comprendre le fonctionnement dans le discours de l'interpellation nous l'analysons dans les échanges. Aussi décelons-nous dans l'interaction didactiquecours de langue plusieurs types d'échanges en fonction du nombre des constituants: i) constitué d'une/deux interventions, ii) deux interventions, iii) trois interventions, iv) plusieurs interventions / \pm plusieurs intervenants.

i) Les échanges constitués de deux interventions suivent la description donnée dans le modèle genevois (Roulet et al., [1985], 1991) : $\mathrm{I}_{1}$ - initiative 爁 $\mathrm{I}_{2}$ - réactive. Mais ce schéma permet plusieurs réalisations en fonction des intervenants, comme nous allons le montrer infra, à l'aide des tableaux.

64 ii) Pour ce qui est des échanges à trois interventions, ceux-ci suivent un schéma très connu qui a été rendu célèbre par Goffman (1974) et repris par presque tous les courants

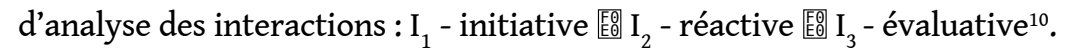

65 À l'intérieur de chaque classe d'échanges on distingue deux niveaux d'analyse - fonction discursive (interactive pour les $\mathrm{I}_{1}$ et $\mathrm{I}_{2}$, et interactionnelle pour la $\mathrm{I}_{3}$ ) et force illocutoire qui correspondent aux unités de rang inférieur, à savoir l'intervention, respectivement 
l'acte de langage. C'est à ce niveau que nous avons « revisité » le modèle genevois. Ainsi, à part le côté « hiérarchique » de ce modèle, ce que l'on retient pour notre approche est directement lié aux notions de fonction et de force. Plus précisément, il s'agit de l'aspect «fonctionnel » de ce modèle. Sauf qu'en ce qui concerne notre réflexion méthodologique, la « fonction (interactionnelle) » est caractéristique de l'intervention tandis que la « force (illocutoire)» est le propre de l'acte de langage. Dans le modèle genevois, la force illocutoire de l'acte nommé « directeur » est transférée à l'intervention. En effet, dans ce modèle il y a deux types d'actes : directeurs (qui sont porteurs de force illocutoires) et subordonnés (qui sont dépourvus de force illocutoire ayant seulement une fonction interactive). C'est ici la différence principale entre le modèle genevois et le nôtre : chaque acte de langage est porteur d'une force illocutoire (au moins). En outre, il n'y a pas de fonction (interactive) entre les actes de langage car nous suivons la description théorique de l'approche du GRC. L'enchaînement conversationnel se fait donc sur les composants de la force illocutoire. Du modèle genevois, on retient aussi les notions de complétude interactionnelle (la contrainte du double accord) et de complétude interactive, nécessaires pour décrire les échanges et les interventions.

Pour rendre l'explication plus claire, nous allons la présenter dans des tableaux d'analyse:

Tableau (1)

\begin{tabular}{|l|l|l|l|l|}
\hline Tour de parole & Intervention & Fonction interactive & Force illocutoire & Intervenant \\
\hline (T1) P & Tu l'as apportée? & initiative (interpellation) & directive & professeur \\
\hline (T2) T & Oui. & Reactive & assertive & élève \\
\hline
\end{tabular}

Tableau (2)

\begin{tabular}{|c|c|c|c|c|}
\hline $\begin{array}{l}\text { Tour de } \\
\text { parole }\end{array}$ & Intervention & Fonction interactive & $\begin{array}{l}\text { Force } \\
\text { illocutoire }\end{array}$ & Intervenant \\
\hline$\left(\mathrm{T}_{1}\right) \mathrm{P}$ & $\begin{array}{l}\text { Ten c'est } \\
\text { combien? }\end{array}$ & $\begin{array}{l}\text { initiative } \\
\text { (interpellation) }\end{array}$ & directive & professeur \\
\hline$\left(\mathrm{T}_{2}\right) \mathrm{E}^{\mathrm{x}}$ & Dix. & Reactive & assertive & élèves \\
\hline$\left(\mathrm{T}_{3}\right) \mathrm{P}$ & Très bien. & Evaluative & expressive & professeur \\
\hline
\end{tabular}

Tableau (3)

\begin{tabular}{|l|l|l|l|l|}
\hline $\begin{array}{l}\text { Tour de } \\
\text { parole }\end{array}$ & Intervention & $\begin{array}{l}\text { Fonction } \\
\text { interactionnelle }\end{array}$ & $\begin{array}{l}\text { Force } \\
\text { illocutoire }\end{array}$ & Intervenant \\
\hline$\left(\mathrm{T}_{1}\right) \mathrm{P}$ & $\begin{array}{l}\text { Quand est-ce que j'ai donné le } \\
\text { travail pour « Le commissaire et } \\
\text { la sorcière »? }\end{array}$ & $\begin{array}{l}\text { initiative } \\
\text { (interpellation) }\end{array}$ & directive & professeur \\
\hline
\end{tabular}




\begin{tabular}{|l|l|l|l|l|}
\hline$\left(\mathrm{T}_{2}\right) \mathrm{E}$ & Avant les vacances. & Reactive & assertive & élève \\
\hline$\left(\mathrm{T}_{3}\right) \mathrm{P}$ & Avant les vacances. & évaluative & assertive & professeur \\
\hline
\end{tabular}

iii) Pour ce qui est de la classe des échanges à plusieurs interventions/ intervenants, elle peut être divisée en deux sous-classes en fonction du nombre de participants: a) échanges à plusieurs interventions/deux intervenants et b) échanges à plusieurs interventions/plusieurs intervenants (voir l'exemple (45)).

Le type d'échange représentatif de l'interaction didactique est celui constitué de trois interventions, la troisième jouant un rôle très important dans la relation didactique professeur-élève. En effet, dans un échange à trois interventions, la $\mathrm{I}_{3}$ ayant une fonction interactive évaluative doublée d'une fonction interactionnelle de clôture est une unité plus complexe que les autres. L'analyse du corpus a mis en évidence le fait que, si la $\mathrm{I}_{3}$ marque une évaluation positive, elle peut être réalisée soit par un acte expressif (comme dans le tableau 2) soit par un assertif (tableau 3). Dans ce cas, la clôture de l'échange ne pose pas de problème et l'interpellation se trouve au niveau de la $\mathrm{I}_{1}$.

Dans ce qui suit nous allons présenter la situation opposée : les évaluations négatives. Ce changement en attire un autre, à savoir l'acte de langage qui correspond à la $\mathrm{I}_{3}$ plus complexe et dont la force illocutoire est souvent difficile à déceler. Dans l'exemple (20), la force illocutoire de cette intervention est directive et cela en fait un échange non " canonique »" . En effet, conformément au " principe de la complétude interactionnelle » propre à l'échange (Roulet et al., 1985), la dernière intervention devrait clôturer l'échange. La fonction de clôture ne peut pas être assurée par un directif «primaire » (Vanderveken, 1988). Il s'agit donc d'un acte de langage « complexe». En effet, un des composants de la force illocutoire est le mode d'accomplissement qui détermine «comment son but doit être accompli sur le contenu propositionnel lors d'un accomplissement d'un acte ayant cette force" (Vanderveken, 1988, 113). Lors d'une demande, par exemple, le locuteur doit donner une option de refus à l'allocutaire. Au contraire, lors d'une demande de justification, comme le cas étudié, le mode d'accomplissement est plus péremptoire et il invoque une position d'autorité sur l'allocutaire, dans ce cas concret, sur l'élève. Un directif qui réalise la troisième intervention d'un échange, qui est en même temps l'intervention qui le clôture, est plus menaçant pour la face de l'interlocuteur que si cet acte se trouvait dans l'intervention initiative. En outre, on remarque une gradation à l'intérieur de la classe des interpellations qui finit dans la dernière par le terme d'adresse qui joue un rôle indexical.

Dans un tel modèle hiérarchique, dans l'interaction didactique, il semble que la distribution privilégiée pour l'interpellation soit dans la première intervention. Toutefois il existe des échanges dans lesquels l'interpellation apparaît explicitement dans la troisième intervention, comme dans l'exemple ci-dessous:

(42) (RLM)

$\left(T_{1}\right)$ P Les fiches tu les as faites?

$\left(\mathrm{T}_{2}\right)$ L Non.

$\left(\mathrm{T}_{3}\right)$ P Comment ça tu les as pas faites? Comment ça, comment ça se fait que tu les aies pas faites, Louis? didactique qui peut être réalisée de quatre manières : 

(i) $\mathrm{E}: \mathrm{I}^{3}: \mathrm{D}-\mathrm{A}-\mathrm{E}^{12}$
(ii) E: $I^{3}: D-A-A$
(iii) $\mathrm{E}: \mathrm{I}^{3}: \mathrm{D}-\mathrm{A}-\mathrm{A}+\mathrm{E}$
(iv) $\mathrm{E}: \mathrm{I}^{3}: \mathrm{D}-\mathrm{A}-\mathrm{CA}$

Le quatrième schéma n'est pas moins spécifique de l'interaction en cours, mais moins fréquent. C'est dans ce schéma que l'interpellation est distribuée deux fois, dans la $\mathrm{I}_{1}$ et dans la $\mathrm{I}_{3}$. En effet, on constate que l'interpellation se combine le plus souvent avec un acte de langage de la classe des directifs.

Les échanges qui finissent par des évaluations négatives qui ouvrent d'autres échanges, en général, s'inscrivent dans le schéma suivant, comme dans l'exemple (42) :

(v) E: : I $^{3}:$ D-A-D

À l'analyse des données des nos corpus, français et roumain, nous remarquons qu'à l'intérieur des échanges il y a deux types de schémas interpellatifs des professeurs: un micro-schème et un macro-schème interpellatif.

a) micro-schème interpellatif didactique :

(43) (FLM)

$\left(T_{1}\right)$ P Est-ce que quelqu'un peut me dire le nombre du mois de décembre requête à adresse collective? ${ }^{13}$

Anne Claire requête à adresse individuelle, tu veux ?

$\left(\mathrm{T}_{2}\right)$ AC Douze.

$\left(\mathrm{T}_{3}\right)$ P Ah, très bien!

La situation est identique en roumain :

(44) (RLM)

$\left(\mathrm{T}_{1}\right) \mathrm{P}$ Ce temă am avut de scris requête à adresse collective ? Florin requête à adresse individuelle.

$\left(\mathrm{T}_{2}\right)$ F Noi am avut tema să scriem propozị̧ii cu ajutorul cuvintelor «livadă »,

«muşuroi ».

$\left(\mathrm{T}_{3}\right)$ P Foarte bine.

(trad.fr.)

$\left(\mathrm{T}_{1}\right) \mathrm{P}$ Qu'est-ce qu'on a eu à préparer comme devoir écrit ? Florin.

$\left(\mathrm{T}_{2}\right) \mathrm{F}$ Nous avons eu à écrire des phrases avec les mots « verger », « fourmilière ».

$\left(\mathrm{T}_{3}\right) \mathrm{P}$ Très bien.

Pour ce qui est de la première intervention, initiative certes, elle est constituée de deux actes de langage :(1) «Est-ce que quelqu'un peut me dire le nombre du mois de décembre? » et (2) « Anne-Claire, tu veux? », les deux étant des directifs. Mais l'on remarque une différence entre les deux actes en ce qui concerne une des composantes de la force illocutoire, à savoir le degré de puissance. Ainsi le degré de puissance des conditions de sincérité du premier acte est plus faible que dans le cas du deuxième acte directif ; et cela parce qu'il a aussi un but illocutoire secondaire de pré-requête.

D'après le modèle d'analyse de GRC, les actes de langage directifs de la première intervention sont réussis et satisfaits lors du deuxième tour de parole, la $\mathrm{I}_{2}$, qui est réactive. C'est grâce à cet acte qu'on a la certitude que le directif a été accompli. En ce sens on cite un fragment de la description du but illocutoire donnée par Vanderveken (1988) «si le locuteur fait une requête ou donne un conseil, le but de son énonciation n'est pas de dire comment les choses sont dans le monde mais bien plutôt de faire transformer le monde par l'action future de l'allocutaire de telle sorte qu'il s'ajuste au contenu propositionnel »(108). 
79 De la même manière, l'acte assertif de l'intervention réactive est accompli avec succès, chose dont on est sûr dans la $\mathrm{I}_{3}$-évaluative. Ici aussi il convient d'étayer ce qu'on vient d'affirmer par un fragment de la description de Vanderveken (idem) « Si le locuteur fait une assertion ou un témoignage, le but de son énonciation est de représenter comme actuel un état de choses et le contenu propositionnel de l'acte de discours est censé correspondre à un état de choses existant (en général indépendamment) dans le monde. De telles énonciations ont la direction d'ajustement des mots aux choses (ou du langage au monde). » On parle, en effet, d'un phénomène de rétrointerprétation.

Vu le nombre élevé d'occurrences dans notre corpus, ce schéma est caractéristique de l'interaction didactique.

81 Une description semblable de ce type d'échange a été présentée d'une part par Y. Soulé et L. Fauré (Praxiling UMR CNRS 5267 / IUFM UM2 et LIRDEF, Montpellier), et de l'autre par R. Bouchard (ICAR, Université de Lyon II) ${ }^{14}$. En effet, ce que nous avons qualifié de "prérequête ", Soulé \& Fauré le décrivent comme " sollicitation collective de la maîtresse ", et ce que nous interprétons comme requête à adresse individuelle, les chercheurs le qualifient de "sollicitation individuelle de la maîtresse». Entre les deux types de descriptions du même phénomène, il n'y a pas de divergence, seulement notre analyse porte en priorité sur le côté linguistique tandis que celle des deux chercheurs susmentionnés vise plutôt le côté didactique. Une raison de plus pour soutenir une interprétation contextualisée de l'interpellation. À son tour, R. Bouchard parle d'un « échange pédagogique » en se référant à ce que nous avons identifié en tant qu'échange à trois interventions. À l'intérieur de cet échange, le chercheur identifie une situation de « polylogue » (maître-élèves) et une autre de « dilogue » (maître-élève). C'est à partir de ces remarques et de nos analyses que nous proposons les deux «schèmes interpellatifs didactiques » mentionnés.

Le deuxième schème est plus étendu :

b) macro-schème interpellatif didactique:

(45) (FLM)

$\left(\mathrm{T}_{1}\right) \mathrm{P}$ Quel ... les news requête à adresse collective . Quitterie. requête à adresse individuelle

$\left(\mathrm{T}_{2}\right)$ Q Le quinze novembre mon papa il s'est opéré il n'en peut plus alors il s'est opéré du nez [...] $]^{15}$

$\left(\mathrm{T}_{3}\right) \mathrm{P}$ Super ! Sixtine. ${ }^{\text {requête à adresse individuelle }}$

$\left(\mathrm{T}_{4}\right) \mathrm{S}$ Hier ma maman elle est allée faire elle a amené ma sœur chez euh chez euh [... ]

$\left(\mathrm{T}_{5}\right)$ P D'accord. Ok. Oui.

$\left(\mathrm{T}_{6}\right)$ E Hier je suis allé au rugby et j'ai gagné une coupe et une médaille [...]

$\left(\mathrm{T}_{7}\right) \mathrm{P}$ Mathieu. requête à adresse individuelle

$\left(\mathrm{T}_{8}\right)$ M Mardi on devait aller au cinéma mais ma mamie était malade donc on n'a pas pu aller au cinéma [...]

(46) (RLM)

$\left(\mathrm{T}_{1}\right) \mathrm{P}$ Toată lumea îmi va citi această cerinŢă. Ia să vedem requête à adresse collective . Flori requête à adresse individuelle

$\left(\mathrm{T}_{2}\right)$ F AlcătuiŢi propoziŢii cu ajutorul cuvintelor livadă, muşuroi.

$\left(\mathrm{T}_{3}\right) \mathrm{P}$ Da. Spune tu prima ${ }^{\text {requête à adresse individuelle. }}$

$\left(\mathrm{T}_{4}\right)$ F Bunica a cumpărat pentru livadă un măr.

$\left(\mathrm{T}_{5}\right)$ P Foarte bună propoziŢia. Altcineva fără să citească cerinŢa requête à adresse collective. Cu cuvântul livadă. Andreea ${ }^{\text {requete à adresse individuelle. }}$

$\left(\mathrm{T}_{6}\right)$ A Pomii din livadă sunt aurii.

$\left(\mathrm{T}_{7}\right)$ P Sunt aurii. [...] 
(trad.fr.)

$\left(\mathrm{T}_{1}\right) \mathrm{P}$ Tout le monde va lire cette consigne. Voyons requête à adresse collective. Flori requête à

adresse individuelle.

$\left(T_{2}\right)$ F Introduisez les mots « verger », « fourmilière » dans des phrases.

$\left(\mathrm{T}_{3}\right) \mathrm{P}$ Oui. Toi la première requête à adresse individuelle.

$\left(\mathrm{T}_{4}\right) \mathrm{F}$ Ma grand-mère a acheté un pommier pour le verger.

$\left(\mathrm{T}_{5}\right) \mathrm{P}$ C'est une très bonne phrase. Quelqu'un d'autre, mais sans lire la consigne

requête à adresse collective. Toujours le mot « verger ». Andreea requête à adresse individuelle

$\left(\mathrm{T}_{6}\right)$ A Les arbres fruitiers du verger sont dorés.

$\left(\mathrm{T}_{7}\right)$ P Sont dorés. [...] fortion-réponse. Dans ce groupe, nous avons choisi de traiter seulement les échanges qui sont formés des réponses peu informatives qui sont presque imposées par la structure des questions - interpellations qui selon (Kerbrat-Orecchioni, 1991) « sollicitent de préférence une réponse positive ou négative tout en laissant malgré tout à L2 la liberté de contrarier cette attente. » (100)

(47)

(T1) P Est-ce que tu crois que quand je donne un travail comme ça ben tous les enfants peuvent le faire quand ils veulent ce travail-là ? Vous croyez ça vous ?

(T2) $\mathrm{E}^{\mathrm{x}}$ Non.

(48)

(T1) P Est-ce que vous avez envie d'apprendre ?

(T2) Ex Oui.

(49)

(T1) P Hm, est-ce que c'est normal de ne pas faire le travail comme ça trois fois ?

(T2) $\mathrm{E}^{\mathrm{x}}$ Non classe, on se pose la question si, de par son statut d'élève, le L2 a vraiment la liberté de contrarier l'attente de L1. Il nous semble qu'avec l'explication de Borillo (1978) - «Le locuteur ne met pas en balance de manière neutre les deux termes de l'alternative. Au contraire, se fondant sur son jugement - qui peut aller de la simple présomption à la quasi-certitude - il se propose selon le degré de sa conviction, de faire confirmer ou de faire admettre son point de vue par la personne à qui il s'adresse, son interlocuteur. Sa stratégie est donc d'orienter celui-ci vers le choix de la valeur que lui-même croit vraie. » (apud Kerbrat-Orecchioni, op.cit., 100) - nous sommes plus proche de la situation étudiée mais pas encore au cœur du sujet. En effet, prenant comme point de départ les deux observations, nous avançons que dans l'interaction didactique, des questions totales comme celles reproduites sous (47), (48) et (49), apparemment alternatives par leur structure sous-jacente, ne laissent pas le choix à L2, sa réponse étant préétablie par toute 
une culture scolaire qu'il partage avec le professeur (L1, dans ce cas) et avec ses camarades. Dans l'interaction didactique les savoirs et les savoir-faire sont plutôt communs au professeur et aux élèves. C'est un cas de dialogisme: le professeur est la «voix» de l'institution. En effet, on pourrait gloser les réponses attendues par des assertions à caractère de postulats extraits du contrat didactique :

$\left(47^{\prime}\right)$ « Les élèves doivent faire le travail dans le délai indiqué par le professeur »

(48') ? « Les élèves ont envie d'apprendre. » plutôt " Nous avons envie d'apprendre »

$\left(49^{\prime}\right)$ «Ce n'est pas normal de ne pas faire le travail trois fois. »

En outre, pour étayer le caractère orienté de la question, nous avons décelé quelques moyens linguistiques, des « orientateurs» (Cf. Kerbrat-Orecchioni (1991) comme le morphème «hm» (exemple (49)), le modalisateur «c'est normal » (même exemple), le présupposé du verbe de la phrase ${ }^{16}$ (exemple (47)) ou la nature du contexte argumentatif (exemple (48)).

\subsection{Autres réalisations de l'interpellation}

Nous avons vu que l'interpellation se réalise en principal par des moyens verbaux qui prennent la forme d'une phrase complète, d'une phrase elliptique ou d'un mot. En outre, nous avons analysé seulement des énoncés qu'on pourrait appeler «classiques " pour l'interpellation. Mais les possibilités de la langue ne sont pas épuisées par rapport aux moyens de réalisations de cet acte. Ainsi mentionnons-nous d'une part l'existence des indices non verbaux et d'autre part des indices mixtes: moyens verbaux standardisés accompagnés des moyens non verbaux.

\subsubsection{Indices non verbaux}

(5/) (RLM)

$\left(\mathrm{T}_{1}\right)$ Dar ariciul ce spune? (fr. « Mais le hérisson, qu'est-ce qu'il dit?»)

$\left(T_{2}\right)$ ((Quelques élèves lèvent le doigt.))

$\left(\mathrm{T}_{3}\right)$ ((Désignation de la tête + la direction du regard.))

91 L'interpellation se trouve au niveau du $\left(\mathrm{T}_{3}\right)$, elle a pour fonction de sélectionner l'allocutaire. On remarque la même situation en français.

92 Par ailleurs, nous postulons que le geste de lever le doigt, dans l'exemple (50), le $\left(\mathrm{T}_{2}\right)$, et dans l'exemple (52) entre $\left(\mathrm{T}_{110}\right)$ et $\left(\mathrm{T}_{111}\right)$, représente une interpellation non verbale, une sollicitation de prendre la parole. On ne peut pas omettre une telle situation, elle était courante dans l'interaction scolaire.

\subsubsection{Indices mixtes}

93 En ce qui concerne cette classe, elle est constitué par des indices verbaux standardisés : le mot « da» (RLM) / « oui » (FLM) a pour fonction d'accorder la parole, accompagné d'un geste (désigner du doigt) ou de la direction du regard. En effet, il s'agit d'une situation hybride, car le mot à lui seul ne pourrait pas fonctionner en tant qu'interpellation sans un indice non-verbal.

(51) (RLM)

P Ce poate să însemne cuvântul «car » ?) Da ? («Quelle est la signification du mot

'char'? Oui ?»)

(52) (FLM)

$\left(\mathrm{T}_{76}\right) \mathrm{P}$ Quel ... les news. requête à adresse collective Quitterie. requête à adresse individuelle

$\left(\mathrm{T}_{77}\right)$ Q Le quinze novembre mon papa il s'est opéré il n'en peut plus alors il s'est 
opéré du nez [...]

$\left(\mathrm{T}_{110}\right)$ P D'accord. Ok.

$\mathrm{E}^{\mathrm{x}}$ ((plusieurs enfants ont levé les doigts)) interpellation non verbale

$\left(\mathrm{T}_{111}\right) \mathrm{P}$ Oui requête à adresse individuelle

$\left(\mathrm{T}_{112}\right)$ E Hier je suis allé au rugby et j'ai gagné une coupe et une médaille [...] l'interpellation. Prenons l'exemple (52) où on a affaire à un macro-schème interpellatif à l'intérieur duquel nous identifions une requête à adresse collective (pré-requête), deux interpellations individuelles et une interpellation non verbale. Ciblons notre attention sur les interpellations individuelles. En effet, comme nous l'avons déjà fait remarquer, il s'agit de deux classes différentes, en dépit du fait qu'elles sont réalisées par des moyens verbaux (au moins partiellement). Elles se différencient surtout par le cotexte, l'enchaînement dans le discours. La première, "Quitterie ", suit une pré-requête qui prépare l'interpellation proprement dite; les deux actes appartiennent au professeur. Tandis que la deuxième " oui », suit une interpellation non verbale qui appartient aux élèves.

\section{Pour une synthèse}

\subsection{Valeurs et fonctions discursives de l'interpellation dans l'interaction didactique}

À travers cette étude nous avons pu constater d'une part l'existence de diverses modalités d'interpellation d'autrui dans l'interaction scolaire, et d'autre part les fonctions que cet acte acquiert dans une interaction authentique.

En ce qui concerne le rapport avec d'autres catégories linguistiques, nous mentionnons tout d'abord que l'interpellation peut être enchâssée dans des actes directifs, assertifs ou expressifs, et que chaque classe d'actes, en fonction du type de phrase, donne à l'interpellation des fonctions différentes. Ainsi rappelons-nous "donner la parole», «sélection du futur destinataire» (acte assertif + modalité assertive/interrogative), « rappel à l'ordre », «faire des reproches » (acte directif + modalité injonctive/assertive/ interrogative), etc.

97 Nous avons vu aussi que l'interpellation peut être «explicitée » par certains indices linguistiques dont nous avons abordé l'un - les TA. Cette unité de la langue en corrélation avec le pronom personnel est en même temps un marqueur de la relation interpersonnelle. Aussi avons-nous vu que le pronom de troisième personne qui correspond au terme qui désigne la personne interpellée est le marqueur de l'interpellation "indirecte", tandis que la deuxième personne rend explicite l'interpellation «directe ». Après l'étude de Goffman (1974) qui établit les notions de « face positive » et « face négative », suivie du travail de Brown \& Levinson (1987), on dira que les TA jouent un rôle essentiel premièrement dans le cadre des stratégies de compensation des FTA (Face Threatening Acts, «Actes menaçant la face»). Aussi mentionnons-nous surtout la présence des interpellations indirectes dans le cadre de l'interaction didactique, les diminutifs, etc. La situation inverse est possible mais nous n'avons pas trouvé d'exemple dans nos corpus ${ }^{17}$. Les TA sont surtout utilisés par les professeurs dans des contextes de FTA afin de ménager les faces des allocutaires - les élèves. 


\section{2. Éléments de synthèse comparative dans le domaine français- roumain}

98 À première vue l'analyse des interpellations met en évidence tellement de similitudes qu'on serait presque tenté de croire à l'existence des « schèmes interpellatifs » identiques dans les interactions didactiques dans les deux systèmes d'enseignement, sauf que ces ressemblances sont dues surtout au contexte situationnel. Aussi l'analyse des interpellations et en particulier des termes d'adresse en emploi appellatif nous montre-telle qu'en ce qui concerne les réalisations linguistiques et la distribution syntaxique, la situation la plus courante est celle où le TA constitue un énoncé à lui seul, et cela dans les deux langues (revoir les exemples (40) et (41)). Cependant, comme nous l'avons démontré dans le corps de la présente étude, aucun terme d'adresse n'existe en dehors de son contexte énonciatif et textuel. Ce type d'énoncé fait partie dans la plupart des cas d'un échange à trois interventions et il est préparé par une pré-requête. D'un autre point de vue, nous mentionnons que ce type d'interpellation, en fonction de la modalité énonciative qui lui est associée, peut exprimer des fonctions différentes dans le discours « donner la parole» (ex. (40 a) et (41 a) ou « demander de se taire» (40 b) et (41 b) - mais il est toujours exprimé à travers un acte ayant une force illocutoire directive. Toujours par rapport à la distribution syntaxique des TA, nous rappelons que les survenues à droite, médiane et à gauche sont présentes dans les deux langues avec les mêmes valeurs discursives que nous avons mentionnées au cours de l'analyse. La similitude la plus importante est l'existence de ce que nous avons appelé «micro- » et «macro-schèmes interpellatifs didactiques" mais cette situation s'explique par le type d'interaction étudiée.

En ce qui concerne les différences entre les deux langues, celles-ci sont plus évidentes au niveau des réalisations linguistiques des interpellations. Par exemple, dans l'interaction didactique roumaine deux traits apparaissent clairement. Premièrement, un nombre important d'énoncés interpellatifs contiennent des interjections injonctives d'exhortation du genre « ia să » ou « hai să » qu'on pourrait traduire en français par «allez $»^{18}$ (ex. (53)). Deuxièmement, nous devons mentionner la fréquence du verbe « dire » en emploi appellatif qui pourrait être glosé par « interpeller » et qui est à la base des énoncés illocutoires directifs qui donnent la parole aux élèves (ex. (54). En français, aucun emploi ne semble apparaître de façon régulière dans ce contexte, du moins dans le corpus étudié.

(53) (RLM) Hai să deschidem să citim lectura « Toamna ». (fr. « Allez, ouvrons donc

le livre pour lire le texte 'l'Automne'.»)

(53) (FLM) (Altă variantă.) Miru, spune. (fr. « (Une autre variante.) Allez, Miru, disnous. ")

Une autre classe de différences se situe au niveau de l'inventaire des TA. On mentionne le nombre important des patronymes associés aux prénoms en roumain; ceux-ci ne jouent pas un rôle d'identification d'un locuteur qui porterait le même prénom qu'un autre, comme c'est le cas en français (situation plutôt exceptionnelle) ${ }^{19}$. Toujours par rapport aux TA, nous signalons la présence des labels en français, situation absente du roumain à cause de l'organisation différente de la classe.

(54) (RLM) Doar răspunsul. Ana Constantin. (fr. '(On va lire) Seulement la réponse.

Anne Constantin.')

(55) (FLM) Les distributeurs ! 
101 En résumé, en dépit d'un nombre important de similitudes entre le roumain et le français, les deux langues ne conçoivent ni la réalisation ni la fréquence des interpellations de la même manière.

\section{En guise de conclusion. Pour une approche " contextualisée » de l'interpellation}

Avec cette analyse du fonctionnement des interpellations dans un corpus d'interactions authentiques, nous avons voulu d'abord mettre en évidence la nécessité de prendre en compte le cadre concret de production de cette unité de la langue. Ensuite, nous avons remarqué que même si les variations de la réalisation des interpellations des professeurs français et roumains sont observables surtout au niveau linguistique, deux autres dimensions s'y ajoutent -culturelle et didactique- pour expliquer ce phénomène complexe qu'est l'interpellation en contexte scolaire. En dernier lieu, il nous semble que c'est la convergence des diverses catégories avec lesquelles l'interpellation interfère, tout juste abordées dans cet article, qui permet le mieux de caractériser ce phénomène. En outre, à la lumière des faits mis en évidence par l'analyse des données authentiques, nous plaidons pour une interprétation contextualisée de l'interpellation.

À la fin, nous voudrions reproduire un extrait du corpus de FLM que nous avons intitulé "Quand l'interpellation interpelle les élèves", qui semble postuler l'idée que l'interpellation est ressentie comme une catégorie naturelle :

(57) (FLM)

P Mais comment on sait que c'est une lettre? Moi je savais. Alors il y avait une signature. Qu'est-ce qu'il y avait d'autre?

E Un mot de politesse.

P Une formule de politesse. C'est bien. Qui peut me donner une formule de politesse ? Qu'est-ce qu'elle écrit sur la lettre ? Oui. ((+ direction du regard))

E Bisous moustachus.

P Bisous moustachus. Qu'est-ce qu'il y avait d'autre? Mahaut?

$M$ Chers enfants.

P Chers enfants. Qu'est-ce que c'est ça ? Comment ça s'appelle?

PA Une interpellation.

P Bravo, Paul Augustin! Interpellation. Ça veut dire quoi interpeller?

PA Ça veut dire qu'elle nous adresse la parole [...]

\section{BIBLIOGRAPHIE}

Araùjo-Carreira, M. H. (sous la dir.) (2006). « Venez, venez ! » De la suggestion à l'injonction dans les langues romanes, Travaux et Documents, 32, Université Paris 8, Vincennes-Saint-Denis.

Benveniste, E. (1974). Problèmes de linguistique générale, Paris : Gallimard.

Brown, P., Levinson, S.C. (1987). Politeness: Some Universals in Language Usage, Cambridge, CUP (2 édition). 
Charaudeau, P. (1992). Grammaire du sens et de l'expression, Paris : Hachette.

Comes, E. (2006). Interjections injonctives d'exhortation en roumain et en français in Araújo Carreira, M.H. (éd.), Travaux et documents, 32-2006, «Venez, venez! » De la suggestion à l'injonction dans les langues romanes, Université Paris 8, Vincennes-Saint-Denis, 75-89.

Kerbrat-Orecchioni, C. (1991). L'acte de question et l'acte d'assertion : opposition discrète ou continuum ?, in Kerbrat-Orecchioni, C. (éd.) La Question. Lyon : PUL.

Kerbrat-Orecchioni, C. (2001). Les actes de langage dans le discours. Théorie et fonctionnement, "Quand dire c'est faire » : un travail de synthèse sur la pragmatique conversationnelle, Paris : Nathan.

Détrie C. (2007). De la non-personne à la personne : l'apostrophe nominale, Paris : CNRS Éditions.

Goffman, E. (1974). Les rites d'interaction, Paris : Les Éditions de Minuit.

Fontaney, L. (1991). À la lumière de l'intonation, in Kerbrat-Orecchioni, C. (éd.) La Question, Lyon : PUL, 113-161.

Lagorgette D. (2006). Du vocatif à l'apostrophe. Problèmes terminologiques et théoriques. Termes d'adresse et détachement en diachronie du français. L'Information grammaticale, 109, 38-44.

Măgureanu, A. (2008). La structure dialogique du discours, București : Editura UniversităŢii din Bucureşti.

Moeschler, J. (1996), Théorie pragmatique et conversationnelle, Paris : Armand Colin.

Neveu F. (2000). Des Noms - Nomination, désignation, interprétations, Paris : SEDES.

Pop L. (2000). Espaces discursifs : pour une représentation des hétérogénéités discursives, Paris : Peeters. Roulet, E. et al. ([1985], 1991), L'Articulation du discours en français contemporain, Berne : Peter Lang, troisième édition.

Rusu, V. (1959). Formules d'interpellation en roumain, Revue roumaine de linguistique IV, 2 :

243-255.

Searle, J. R. (1969/1972). Les actes de langage, Paris : Hermann.

Searle, J. R. (1979/1982) : Sens et expression, Paris : Éditions de Minuit.

Searle, J. R., Vanderveken, D. (1985). Foundations of Illocutionary Logic, Cambridge: Cambridge University Press.

Trognon, A., Brassac, C. (1992). L'enchaînement conversationnel, Cahiers de Linguistique Française, 13, 76-107.

Vanderveken, D. (1988). Les actes de discours, Liège : Pierre Mardaga.

Vanderveken, D. (1992). La théorie des actes de discours et l'analyse de la conversation, Cahiers de Linguistique Française, 13, 9-61.

\section{NOTES}

1. L'auteur renvoie, sur ce point, à la littérature consacrée à ce sujet : tout d'abord, Searle $(1979 / 1982,58)$ notait que des actes tels que " définir ", " abréger ", " appeler ", " surnommer ", etc., appartenant à la classe des déclaratifs, n'exigent pas la présence d'une institution extralinguistique grâce à la propriété qu'auraient ces actes à "concerner le langage même " (ibidem); d'autre part, parmi les actes représentatifs Searle en identifie quelques-uns qui 
auraient un comportement syntaxique différent des autres énoncés de cette classe : il s'agit d'actes tels " diagnostiquer ", " appeler ", " décrire ", " classer ", " classifier ", " identifier ", mais Searle renonce à en constituer une classe à part. Enfin, Katz (1977) parle d'actes de nomination - «nommer ", « baptiser », « appeler », « surnommer » - dont le but est d'assigner à jamais un nom à un récepteur (cf. Măgureanu, 2008, 268).

2. Pour une analyse complète, on devrait parler des marqueurs de nature prosodique et typographiques. Même si dans nos transcriptions nous utilisons des signes conventionnels susceptibles de mieux décrire les phénomènes oraux, pour cet article, nous avons choisi de mettre des signes de ponctuation pour rendre les exemples plus «lisibles». Toutefois, nous allons utiliser quelques sigles comme: RLM pour « roumain langue maternelle» et FLM pour «français langue maternelle», P pour "professeur », E pour «élève», Ex pour «la classe d'élèves », T $(1,2 \ldots \mathrm{n})$ pour « tour de parole », l'initiale majuscule du nom de l'élève s'il est révélé dans le discours.

3. L'objet d'étude de cet article n'étant pas les actes de langage, nous avons choisi de donner une description succincte, sans nous attarder sur des cas litigieux, comme par exemple le cas de la coexistence de plusieurs actes de langage dans le même énoncé, ce que Fontaney (1991) appelle « superposition d'actes» (157).

4. Ces deux acceptions du terme ne s'excluent pas réciproquement en dépit des polémiques plutôt stériles qui ont eu lieu entre les tenants de la théorie des speech acts d'un côté et ceux de l'analyse du discours de l'autre. Pour plus de détails par rapport aux positions théoriques différentes de Searle et de Moeschler en ce qui concerne les actes de langage, voir Moeschler, J. (1996, pp.195-206), mais aussi Brassac (1992).

5. En réalité, c'est la même description que celle de Kerbrat-Orecchioni (2001 : 91), à la seule différence que nous considérons que les actes à accomplir par l'interlocuteur peuvent être aussi de nature verbale. En ce qui concerne ce type de réaction à une requête, nous mentionnons que celle-ci ne constitue pas un apport d'information comme dans le cas de la réponse à une question.

6. Dans cet exemple nous avons affaire à deux termes qui désignent les interpellés, Alexandra (+ 3e personne) et copii (fr. « les enfants »+2e personne), mais nous nous intéressons ici seulement à celui corrélé avec la $3 e$ personne.

7. Conformément à l'approche de l'enchaînement conversationnel du GRC de Nancy 2 que nous avons présenté supra.

8. Vu la nature de notre corpus et le type d'approche proposé, nous utiliserons la terminologie spécifique de l'analyse des conversations, à savoir «terme d'adresse ", soit TA pour un référent au singulier et TAs pour le pluriel.

9. Le nom rappelle le célèbre personnage de la période de l'Empire Romain, Brutus, le fils de César, celui qui finit par le tuer. Dans l'exemple cité, il s'agit d'un emploi ironique motivé par la situation concrète. En effet, l'élève surnommé « Brutus » arrive en retard, comme d'autres élèves d'ailleurs, sauf que lui est un élève «modèle " par rapport aux exigences scolaires. Cette situation est une preuve de plus que l'interprétation de certaines unités linguistiques ne peut pas être séparée de son contexte.

10. La troisième intervention de ce type d'échange appartient au professeur en général, ayant une double fonction : d'évaluation (fonction interactive) et de clôture (fonction interactionnelle). Du point de vue interactif, les actes font partie des interventions qui sont liées et qui forment un échange conformément au "principe de la complétude interactionnelle » (la contrainte du double accord de Roulet et al., 1985).

11. Dans le sens de « majoritaire » dans l'interaction didactique. Cela ne veut pas dire que ce type d'échange n'est pas spécifique de ce type d'interaction. Au contraire. 
12. $\mathrm{E}: \mathrm{I} 3=$ échange à trois interventions $; \mathrm{I} 1 / 2 / 3=$ première/ deuxième/troisième intervention ; $\mathrm{D}$ $=$ directif $; \mathrm{A}=$ assertif $; \mathrm{E}=$ expressif $; \mathrm{A}+\mathrm{E}=$ addition d'actes $:$ un assertif suivi d'un expressif $; \mathrm{CA}=$ cumul d'actes ou « superposition d'actes » (Fontaney, 1991)

13. Le premier acte de langage a la force illocutoire de requête, mais dans ce contexte il acquiert plutôt une force de pré-requête, d'après les termes de Levinson (apud Kerbrat-Orecchioni, 2001). Et cela justement parce que l'interpellation n'a pas d'adresse directe.

14. Il s'agit des communications présentées dans le cadre du colloque L'Interpellation. Perspectives linguistiques et didactiques (16-17 mai 2008). Pour plus de détails, voir les articles de ces auteurs mentionnés dans ce même numéro thématique.

15. Faute d'espace nous n'allons pas reproduire intégralement les discours des élèves.

16. Le verbe "croire" dans cet exemple est un contre-factif, il oriente donc la question dans un sens négatif.

17. Toutefois on mentionne l'usage des noms de famille dans l'interaction en classe de RLM associé à des actes directifs de recadrage. Dans cette situation le TA est plutôt un marqueur de FTA qu'un compensateur de l'agression des faces de l'allocutaire.

18. L'étude de Comes (2006) donne une image très claire de cette situation.

19. On postule que cette différence est due au contexte historique et culturel. Avant 1989 il était courant dans toutes les institutions d'utiliser le patronyme pour nommer les personnes. La situation relevée dans ce corpus pourrait être témoin d'une réminiscence de cette facture.

\section{RÉSUMÉS}

L'interpellation interpelle les chercheurs étant un phénomène qui rend surtout compte d'un flou catégoriel. Ce flou vient vraisemblablement du fait que cette unité recoupe plusieurs catégories linguistiques comme les vocatifs, les pronoms personnels, les termes d'adresse, les injonctions, les actes de langage, etc. Au lieu de s'épuiser en cherchant des critères d'analyse qui relèvent d'un domaine ou d'un autre ou d'essayer de donner une définition unitaire à un phénomène si complexe - que nous traitons comme acte métadiscursif - il vaut mieux prendre en compte les catégories avec lesquelles elle entre en contact. C'est pour cette raison que nous proposons une approche sociopragmatique de l'interpellation en lien avec les catégories mentionnées supra et cela dans le but principal de lui donner une description fonctionnelle. À ce but vient s'en ajouter un autre, plus particulier, celui d'étudier les réalisations linguistiques des interpellations en français et en roumain en tant que langues maternelles. L'observation des données issues de deux corpus constitués d'enregistrements des classes de langue maternelle, français et roumain, a permis de donner un nouvel éclairage à la question de l'interpellation en milieu scolaire et de mettre au jour l'existence des micro- et des macro-schèmes interpellatifs communs aux deux langues étudiées. En ce qui concerne les différences, celles-ci relèvent du domaine historicoculturel ou didactique, comme par exemple l'existence d'un grand nombre de patronymes en roumain pour désigner les interpellés, tandis qu'en français on mentionne les labels pour le même phénomène.

The Interpellation challenges the researchers, as it is a phenomenon which is in touch with a vague domain. It is so vague probably because its origins reside in its relatedness to several linguistic categories, such as vocatives, personal pronouns, forms of address, injunctions, speech acts, etc. Instead of striving to understand the meaning of such a complex phenomenon, by 
looking for strict criteria to define this, it is better to concentrate our attention on these related facts which are always in contact with it. We embrace the idea that the interpellation is a metadiscursive act. Considering that, we propose a sociopragmatical approach to analyze interpellation, taking into account the above mentioned categories, thus giving a functional description to this act. In addition to this we are also studying the linguistic performances which appear in Romanian and French as native languages. The observation of naturally data which we collected by recording native language classes, French and Romanian, shows a new path to describe interpellation in student environment and on the other hand we submit to the general attention the existence of micro- and macro-interpellative schemes which are common for the both mentioned languages. The differences consist, for example, in a large amount of patronymic forms of address in Romanian, whereas in French we observed the "labels" for the same phenomenon, all this due to historical, cultural and didactical context specific to each type of interaction.

\section{INDEX}

Mots-clés : acte métadiscursif, interaction didactique, interpellation, schèmes interpellatifs, terme d'adresse

Keywords : didactic interaction, forms of address, interpellative schemes, metadiscursive act

\section{AUTEUR}

\section{VALENTINA BARBU}

Université de Bucarest, Université de Provence (Centre d'Aix), LPL 